\title{
A Sense beyond All Senses: That Sense of Errors Changing Our Lives in the Technology Driven World
}

\author{
Debatri Das
}

\begin{abstract}
We all have probably generated this wish since the time of being a kid and while dealing with an wild guess in a quiz competition, we all used to think what if I would develop that sense within us that would lead us towards the correct answer. The question here arrives is, how we would mend through this system to make one non erroneous sense.
\end{abstract}

\section{INTRODUCTION}

There is a common knowledge goes that human beings have five basic senses which are touch, sight, hearing, smell and taste. The sensing organs mentioned above are associated with particular senses send information to our brain (through multiple motor sensors) to help us understand the world vividly around us. But there are the possibilities of other senses as well and of course we cannot deny that fact.

And thinking of that extra sense, it takes me a great deal of wonder what if we have that sense to pre check every error that occurred by us and get it corrected. Seems nothing new, isn't it?

Let me give you an example. What if we have a certain destination and the path is unfortunately unknown. Then we are left with only one option which is to start dealing with an unknown road .And here comes my wish. I want the path to be optimally accurate which means the probability of erroneousness should be 0 or nearly 0.I wish for a sense which dictates the right or accurate path so that we do not need to depend on guts or guess. Call it whatever you prefer.

\section{SYSTEM DESCRIPTION}

I am trying to talk about such a sensor that would be interfaced between human and machine. The machine has to be completed with feeding with the knowledge of learning and working including an object of better performance and some accurate visualization ability. That is the power of exponential growth, and today nanotechnology and Artificial Intelligence is bound on that path. Machine Learning and Optimization are some of the manifesting aspects that drive Artificial Intelligence along with the help of micro electronics systems.

Here I am discussing about an intelligent communication system in a form of nano sensor on the basis of different coding techniques including convolutional coding, trellis mechanism and along with the help of bellman equation The study of such a system with various coding techniques can be represented by Petri net model for better understanding of how the system works depending on the action, state and the reward gained after probable and justified action. Let's have a look on how the model looks:

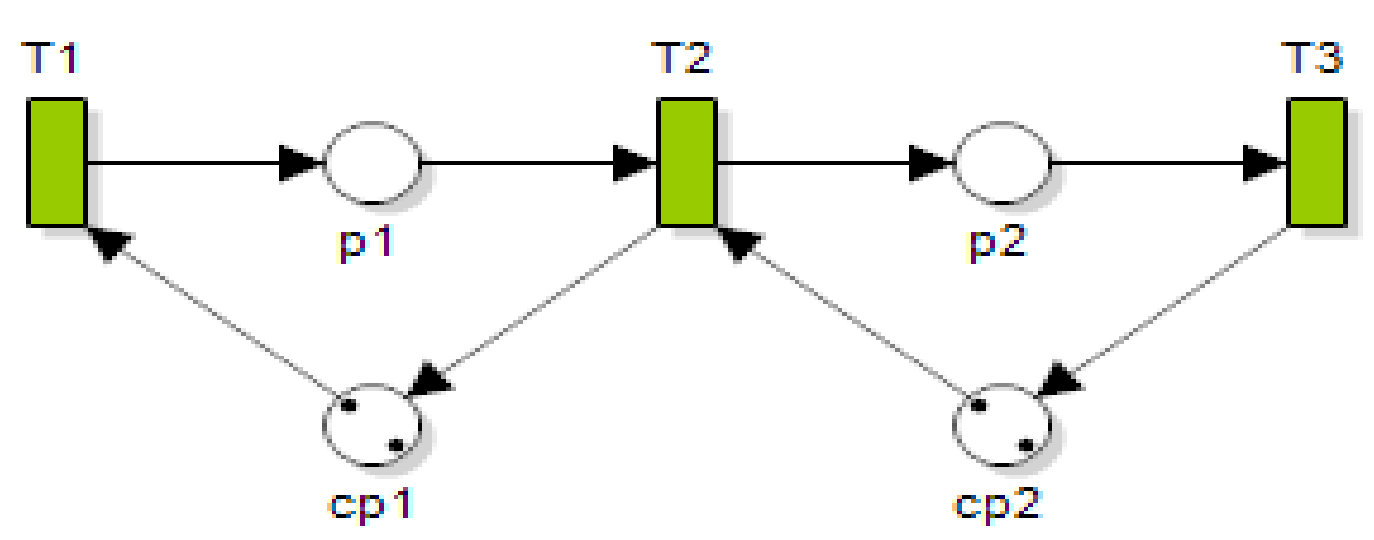

Fig 1: Petri net Model

T1, T2, T3: states

p1, p2: actions

cp1,cp2: Interconnected nodes referring to rewards.
For the better understanding of the probability factor associated with it, we can refer to bellman action which makes a more clear idea regarding the petri net model. 


\section{$>$ What is Petri Net model?}

Petri net models, also known as a place/transition (PT) net, is one of the most reliable mathematical modeling languages for the description of distributed systems that depends on randomness or stochastic. It is a class which is based on the event dynamic system, which are discrete in nature. We can call a Petri net as a directed bipartite graph, in which the nodes represent transitions (sometimes represented by bars) and places or conditions, represented by circles. The directed arcs describe which places are before and/or after situations or let us say conditions, specifically. Some sources state that Carl Adam is the inventor of Petri net for the purpose of describing chemical processes in the year 1939. Like industry standards such as UML activity diagrams, Notation and event-driven process chains, Business Process Model, Algebraic equations Petri nets offer a graphical notation for stepwise processes that include choice, action, decaying factors, iteration, and simultaneous execution.

\section{Trellis Mechanism:}

Trellis displays a certain framework that approaches towards the visualization of a particular set of data. For example, we can say that Trellis display depends on a strong technique for understanding the exchange study about how a response depends on different variables which is to be explained. With the help of trellis mechanism we can have an understanding of how changing the code rate can affect the ability of the system to correct errors. The trellis mechanism along with the maximum likelihood method will work on the high performance error correction technique.

A trellis is basically a graph that has nodes in such a order that the vertical slices with each node at one time is inter linked to one node(minimum) at before and at least one node at an after execution time.

Trellises are used in several encoders and decoders, also in the concatenated decoders for communication theory and encryption that is based on the maximum likelihood method. Let us see how trellis mechanism works:

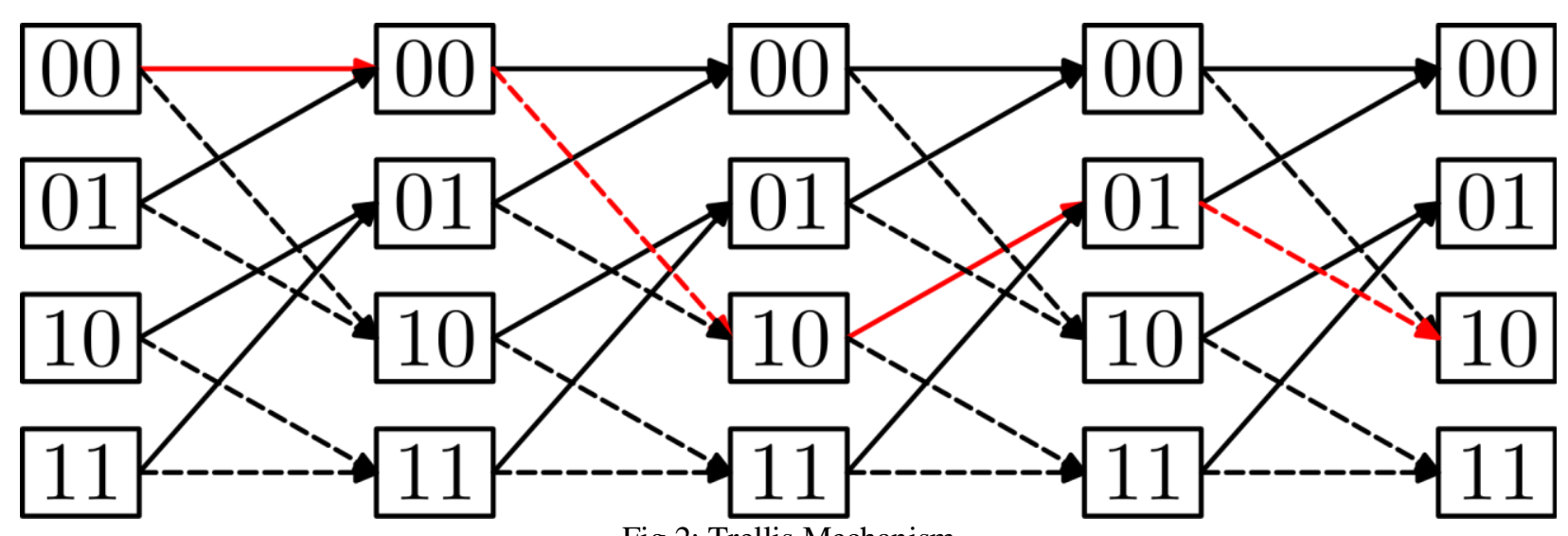

Fig 2: Trellis Mechanism

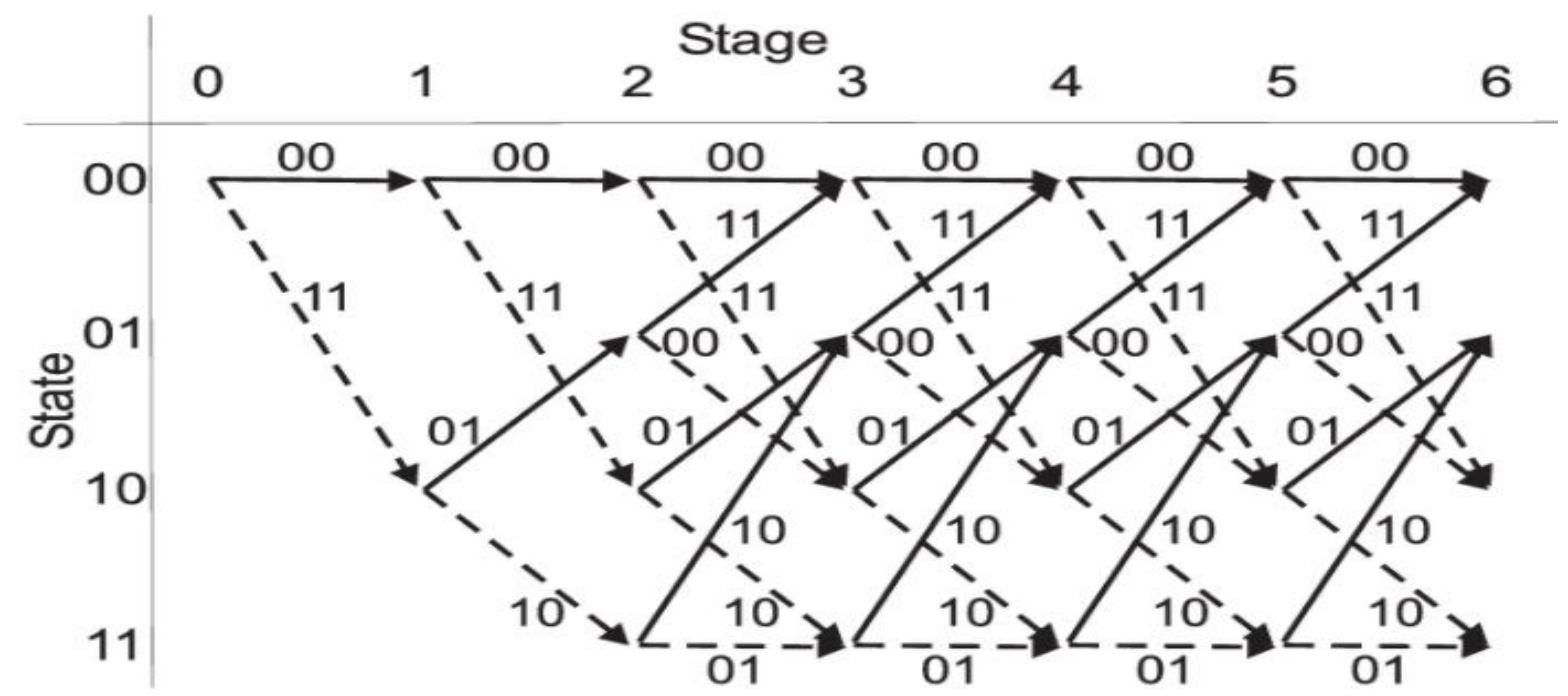

Fig 3: Viterbi Algorithm applied on multi stated Trellis 


\section{Basic Convolution Coder Implementation}

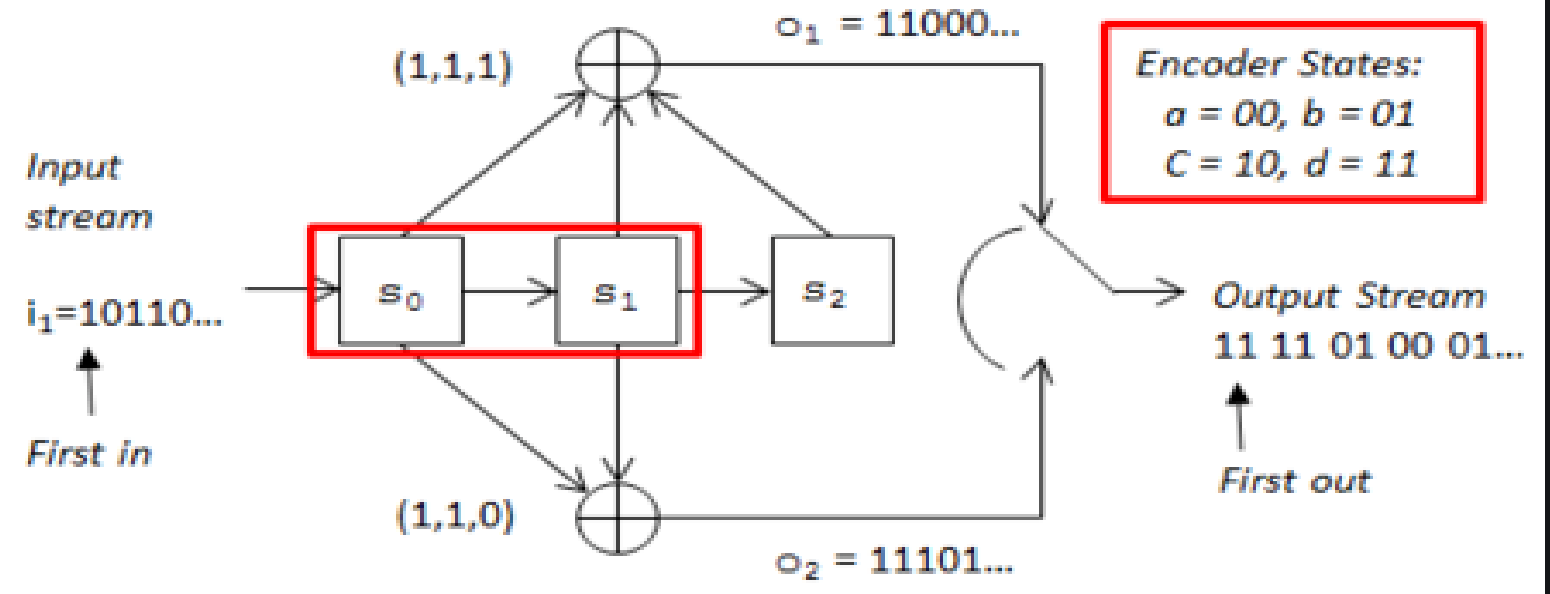

Coder Rate $1 / 2$, and configuration $(n, k, m)=(2,1,3)$ where:

$n$ : is the number of output bits $k$ : is the number of input bits

$m:$ is the number of shift register stages

Fig 4: Code rate implementation in a convolution Encoder

\begin{tabular}{|c|cccc|c|c|}
\hline $\begin{array}{c}\text { Memory } \\
\nu\end{array}$ & \multicolumn{7}{|c|}{$\begin{array}{c}\text { Generation in } \\
\text { octal notation }\end{array}$} & $\begin{array}{c}\text { Upper bound } \\
\text { on } d_{e}\end{array}$ \\
\hline 1 & 1 & 1 & 3 & 3 & 6 & 6 \\
2 & 5 & 5 & 7 & 7 & 10 & 10 \\
3 & 13 & 13 & 15 & 17 & 13 & 15 \\
4 & 25 & 27 & 33 & 37 & 16 & 16 \\
5 & 45 & 53 & 67 & 77 & 18 & 18 \\
6 & 117 & 127 & 155 & 171 & 20 & 20 \\
7 & 257 & 311 & 337 & 355 & 22 & 22 \\
8 & 533 & 575 & 647 & 711 & 24 & 24 \\
9 & 1173 & 1325 & 1467 & 1751 & 27 & 27 \\
10 & 2387 & 2353 & 2671 & 3175 & 29 & 29 \\
11 & 4767 & 5723 & 6265 & 7455 & 32 & 32 \\
12 & 11145 & 12477 & 15537 & 16727 & 33 & 33 \\
13 & 21113 & 23175 & 35527 & 35537 & 36 & 36 \\
\hline
\end{tabular}

Fig 5: Convergence of concatenated Codes based on upper bounds

Here, from the above picture we get an idea about the analysis of the convergence of the convolution code is described through parametric initialization study approaches. The codes, contain high rates of performance regarding the error correction techniques. Trellis mechanism helps to show how varying a code rate will disturb the ability of correcting errors in codes.

For an example, when a binary image is encoded by a convolution encoder before transmission and decoded after reception, there is a chance to get the same image no matter even if we use concatenated convolution coding method. This powerful tool of generating function analysis is demonstrated through petri net models to yield the arbitrary codes for gaining both upper bounds and distance properties of the bit error rate probability for any memory less channel.

\section{- Enhancing Stability Criterion:}

After we generate the high performance code rate for the errorless system using such coding technique, we need to focus on the stability criterion of the system, which would make the system stable. Now another problem arises regarding achieving the stability criterion on this system. And to achieve that, we can opt for the approach one of the most important section of artificial intelligence which is reinforcement learning or Q learning. Q learning is one of the technique which are under Reinforcement learning in machine learning(ML) . This area of machine learning is concerned with how the agents should take actions in an environment in order to gain the maximum reward.

Therefore, we can derive that Q learning based petri net models provide boundary conditions and limits for convolution codes to act efficiently. 
ISSN No:-2456-2165

The Q learning mechanism provides an idea of the higher learning rate, the sense of developing better reward gain of the agent, and lower temporal difference and the parametric changes and the interconnections helps to select the favorable path of a system to follow for the agent for better efficiency.

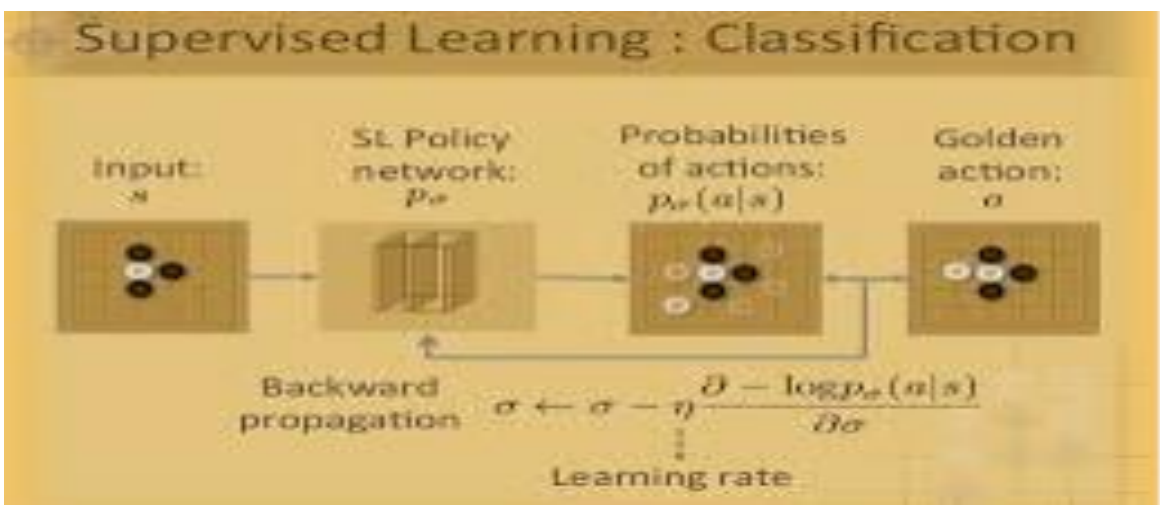

Fig 6. The Learning Process

$$
Q^{\text {new }}\left(s_{t}, a_{t}\right) \leftarrow \underbrace{Q\left(s_{t}, a_{t}\right)}_{\text {old value }}+\underbrace{\alpha}_{\text {learning rate }} \cdot \overbrace{\underbrace{\underbrace{r_{t}}_{\text {reward }}+\underbrace{\gamma}_{\text {discount factor }} \cdot \underbrace{\max _{a} Q\left(s_{t+1}, a\right)}_{\text {estimate of optimal future value }}}_{\text {new value (temporal difference target) }}-\underbrace{Q\left(s_{t}, a_{t}\right)}_{\text {old value }})}^{\text {temporal difference }}
$$

Fig 7: Q learning algorithm

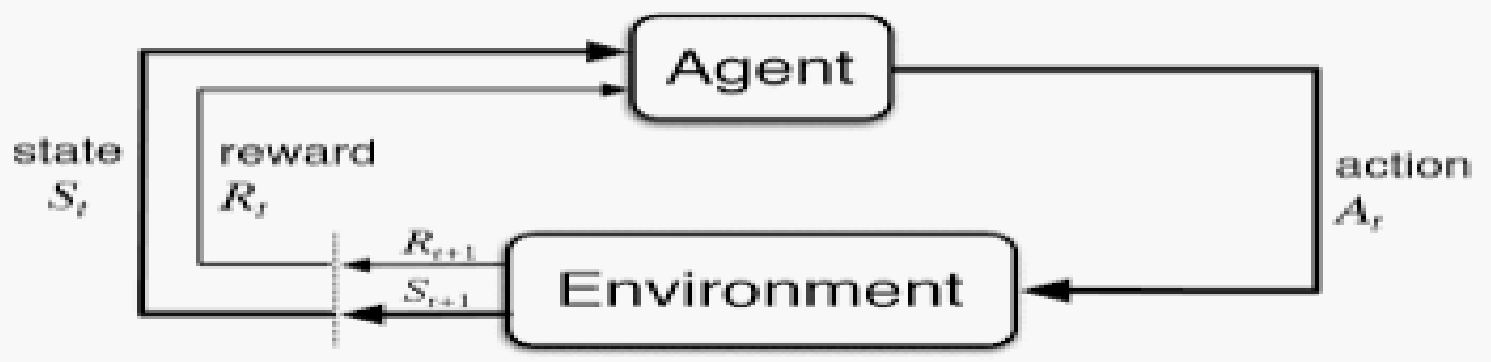

Fig 8: Q Learning Operation

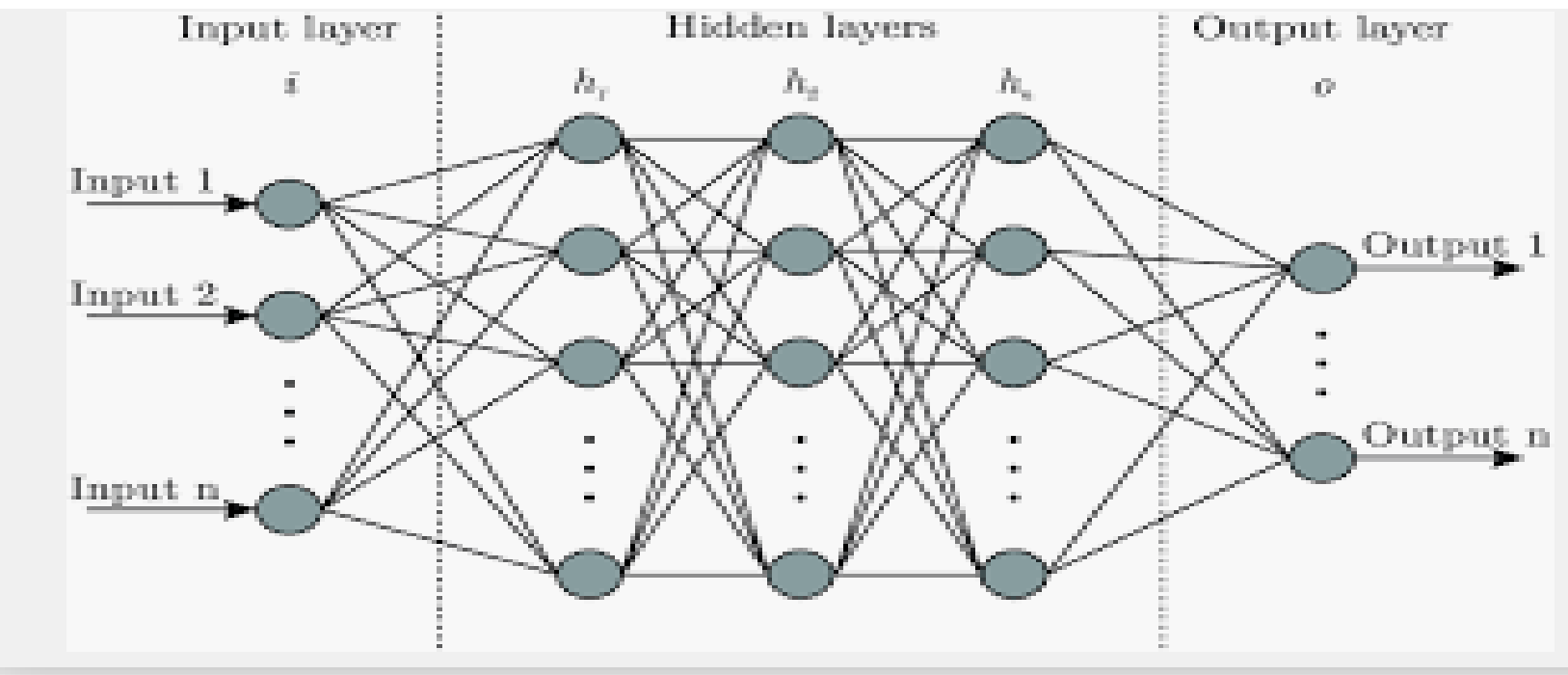

Fig 9: Artificial Neural Network 


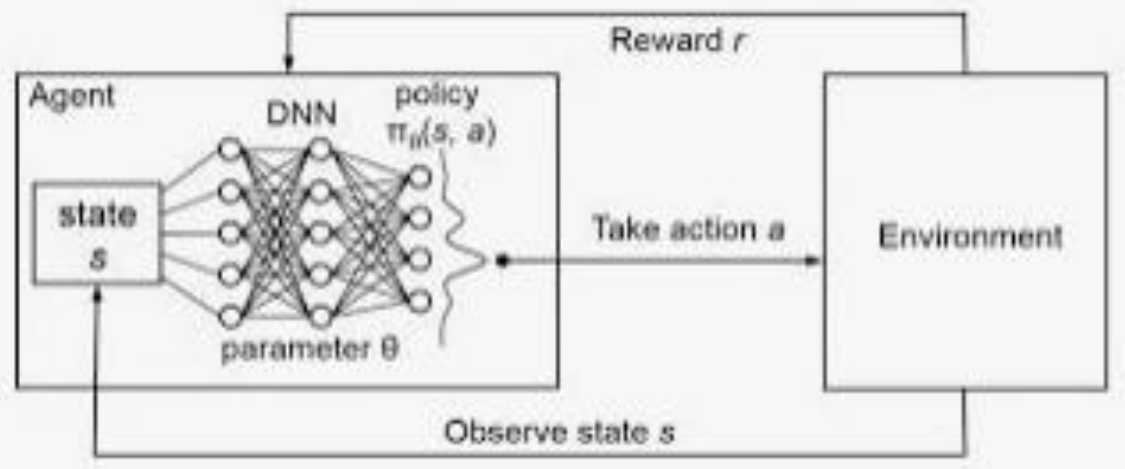

Fig 10: The System sensing errors
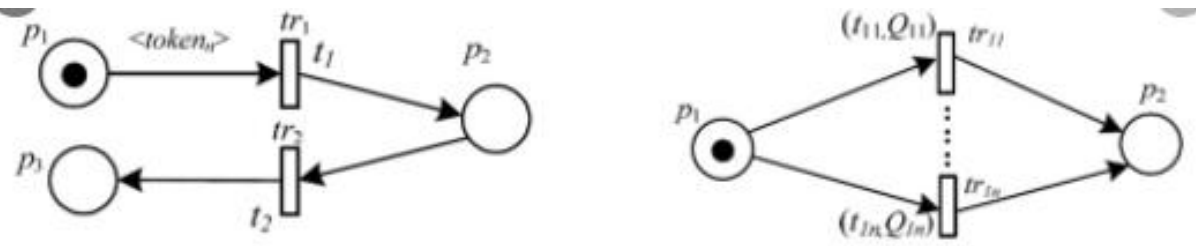

(i) The high-level time Petri net model (ii)The discretization learning model for the delay time

Fig 11: Q learning based Petri net

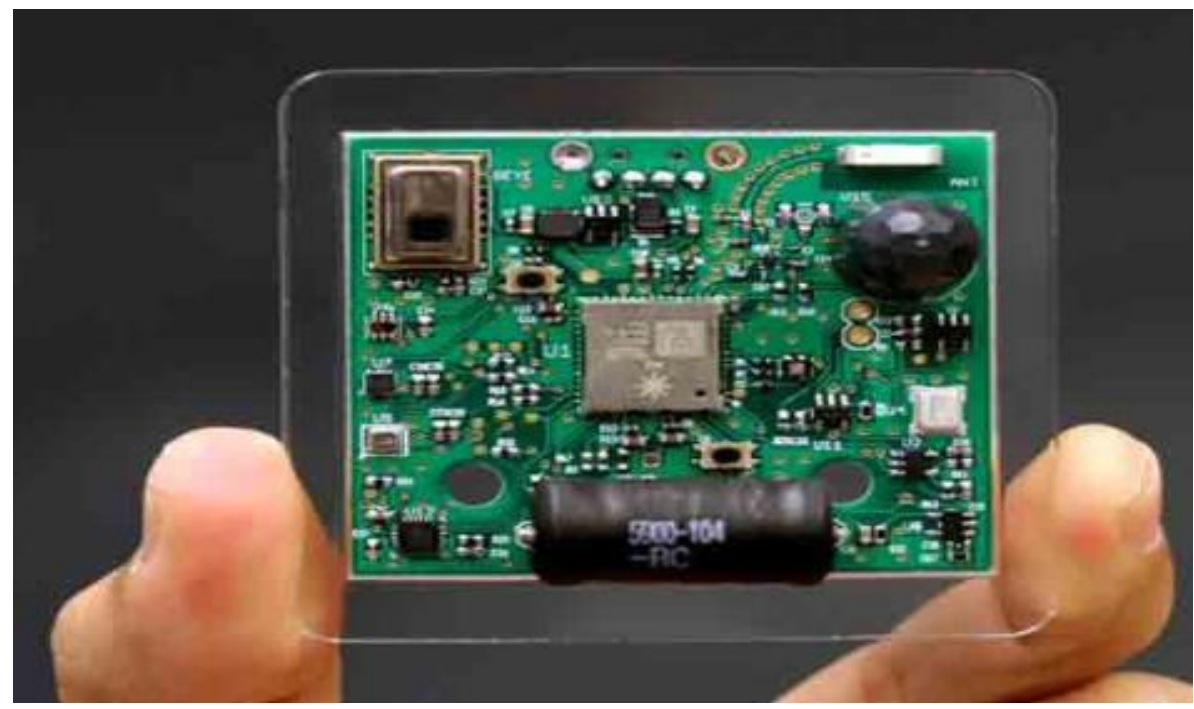

Fig 12: AI Sensor

\section{SCOPE}

In $\mathrm{Q}$ learning based petri net model we can provide boundary conditions and limit for convolution code to act efficiently.

In $\mathrm{Q}$ learning mechanism, the study of improving learning rate, better reward gain, by the agent, lower temporal difference and the parametric interconnections helps to select the favorable path for the system to follow for the agent so that it achieves efficiency. This system thus, implemented as a sensor, not only achieves productivity but also increase high quality and flexibility. And the idea is to implement this into such a nano bio sensor or in today's perspective an AI sensor to act non erroneously in order to achieve maximum reward. This is how the formation of creating a sensor works under the view of IOT.

\section{CONCLUSION}

This paper tells us about the intelligent cooperative communication which is based on different coding techniques including convolution decoding, encoding, viterbi algorithm, maximum likelihood technique along with petri net model representation. The $\mathrm{Q}$ learning mechanism provides an idea of the higher learning rate, the sense of developing better reward gain of the agent, and lower temporal difference and the parametric changes and the interconnections helps to select the favorable path of a 
system to follow for the agent to ameliorate efficiency. The intelligent system increase productivity, along with insuring high stability, flexibility, upgraded quality , Accuracy in performance and lastly, higher safety for a successful industrial measure.

\section{REFERENCES}

[1]. A Theoretical Analysis of Deep Q-Learning, A. Bayen, A. Jadbabaie, G. J. Pappas, P. Parrilo, B. Recht, C. Tomlin, M.Zeilinger.

[2]. Jens Kober and Jan Peters. Reinforcement learning in robotics: A survey. In Reinforcement Learning, pages 579-610. Springer, 2012.

[3]. Christopher JCH Watkins and Peter Dayan. Qlearning. Machine learning, 8(3-4):279-292, 1992 